\section{DESIGN AND APPLICATION OF GROUNDED PIN-PAD RESONATORS IN LTCC COMPONENTS}

\section{K. Rambabu and J. Bornemann}

Department of Electrical and Computer Engineering

University of Victoria

Victoria, BC, Canada V8W 3P6

Received 21 May 2005

ABSTRACT: An equivalent-circuit model for pin-pad resonators is presented. Over a range of substrate permittivities between 2.2 and 35, the model is shown to provide fast and accurate results for series and parallel resonance frequencies. The model is then applied to create transmission zeros in and suppress the harmonics of an LTCC filter structure. This approach is verified by comparison with the results obtained using commercially available software. (C) 2005 Wiley Periodicals, Inc. Microwave Opt Technol Lett 47: 321-323, 2005; Published online in Wiley InterScience (www.interscience.wiley.com). DOI 10.1002/mop. 21158

Key words: pin-pad resonators; LTCC technology; equivalent circuits, filter design

\section{INTRODUCTION}

Pin-pad resonators are formed by metal patches, which are grounded by via holes. As an array, they are widely used to create electromagnetic bandgaps (EBG), for example, [1], where the individual pin-pad component represents the unit element in an EBG structure. Pin-pads have been explored as slow-wave structures in the miniaturization of patch antennas and in delay lines [2]. The filtering effect of these grounded patches embedded in a microstrip-line substrate was studied in [3]. Although pin-pad structures find many applications in various technologies, and especially in LTCC circuitry, component design is plagued by the lack of proper analytical methods.

Therefore, this paper proposes an equivalent-circuit model for pin-pad resonators. Based on well-known empirical expressions for the circuit elements, formulas for the resonating frequencies are derived. The results are then applied to pin-pad resonators within an LTCC filter where they create transmission zeros and/or suppress harmonic passbands of the filter.

\section{THEORY}

Figure 1 shows the pin-pad resonator and a microstrip line in a multilayered circuit environment. The electromagnetic interaction between the pad and the line as well as that between the pad and ground are predominantly capacitive. The grounding pin (via hole) can be modeled as an inductor. Thus, the equivalent circuit is obtained, as shown in Figure 2.

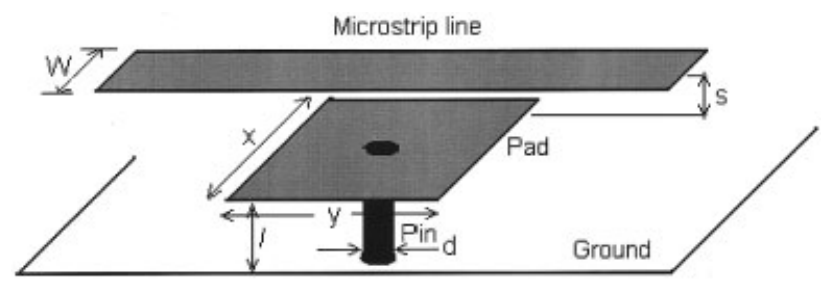

Figure 1 Schematic diagram of a pin-pad resonator coupled to a microstrip line

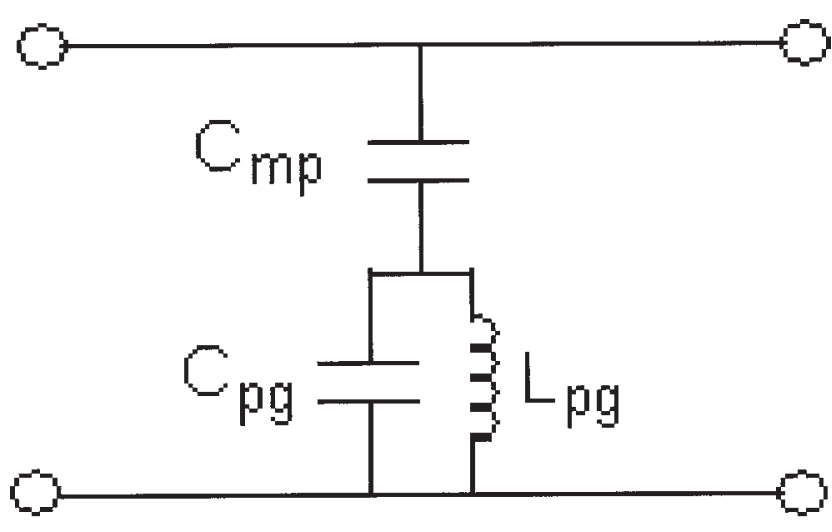

Figure 2 Equivalent-circuit model of the pin-pad resonator coupled to a microstrip line

Empirical expressions, for example, those reported in [4], can now be used to compute the equivalent circuit's element values. Assuming that all physical dimensions (see Fig. 1) are given in meters, then the pin inductance (in Henry) is given by

$$
L_{p g}=\frac{\mu_{0}}{2 \pi} l\left[\ln \left(\frac{4 l}{d}\right)+0.5\left(\frac{d}{l}\right)-0.75\right] .
$$

The pad-to-ground capacitance (in Farad) are computed from

$$
C_{p g}=\frac{\varepsilon_{r} \varepsilon_{0}(x+\Delta p)(y+\Delta p)}{l},
$$

where

$$
\Delta p=\frac{4 l \ln (2)}{\pi} .
$$

To calculate the microstrip-to-pad capacitance (in Farad), two cases are treated separately (see Fig. 1 for quantities $W$ and $x$ ) as follows:

$$
\text { If } W \leq x \text {, then } C_{m p}=\frac{\varepsilon_{r} \varepsilon_{0} W_{e f f}(y+\Delta s)}{s},
$$

where

$$
\Delta s=\frac{4 s \ln (2)}{\pi}
$$

and $W_{\text {eff }}$ is the effective width of the microstrip line (see, for example, [4]).

$$
\text { If } W>x \text {, then } C_{m p}=\frac{\varepsilon_{r} \varepsilon_{0}(x+\Delta s)(y+\Delta s)}{s},
$$

with $\Delta s$ given in Eq. (3b).

With reference to Figure 2, it is obvious that there will be two resonances. One is a series resonance between the terminals and the other is the parallel resonance of the lower loop. Therefore, the structure behaves as a short circuit at the series resonance, given by

$$
f_{s}=\frac{1}{2 \pi \sqrt{L_{p g}\left(C_{m p}+C_{p g}\right)}},
$$


TABLE 1 Permittivities and Dimensions in $\mathrm{mm}$ of the Three Cases Investigated (See Also Fig. 1)

\begin{tabular}{lllc}
\hline Dimensions & Case 1 & Case 2 & Case 3 \\
\hline$\varepsilon_{r}$ & 2.2 & 7.5 & 35 \\
$W$ & 2.46 & 0.6 & 0.1 \\
$h=s+l$ & 0.8 & 0.5 & 0.5 \\
$l$ & 0.4 & 0.375 & 0.3 \\
$d$ & 0.2 & 0.2 & 0.1 \\
\hline
\end{tabular}

and as an open circuit at the parallel resonance, given by

$$
f_{p}=\frac{1}{2 \pi \sqrt{L_{p g} C_{p g}}} .
$$

In order to validate the equations presented above, the resonance frequencies for three different geometries and substrate permittivities (see also Table I) are plotted in Figures 3 and 4 as a function of the side length of the square pads $(x=y)$. Also shown are the respective values obtained using the commercial full-wave analysis tool IE3D ${ }^{\odot}$. Both the series (Fig. 3) and the parallel (Fig. 4 ) resonances display very good agreement with the IE3D ${ }^{\odot}$ results. Note that the series resonances, which are usually employed to create transmission zeros, are in better agreement with the fieldsolver results. Also note that the parallel resonances for case 3 in Figure 4 fall outside the range of practical applications and, therefore, are not shown.

\section{APPLICATION}

The stopband performance of the bandpass filters can be improved by including more resonators, for example, [5]; higher-order harmonics are usually eliminated by a following lowpass filter (see, for example, [6]). LTCC technology is built on multilayered circuitry where the relatively high permittivity and the vertical dimension are effectively used to miniaturize the component. In [7], a coupling pad beneath the resonators is used to create a transmission zero in LTCC filters. A similar arrangement with two resonators and input/output coupling can create two transmission zeros (see, for example, [8]), but at the expense of the transmission zeros not being independently controlled.

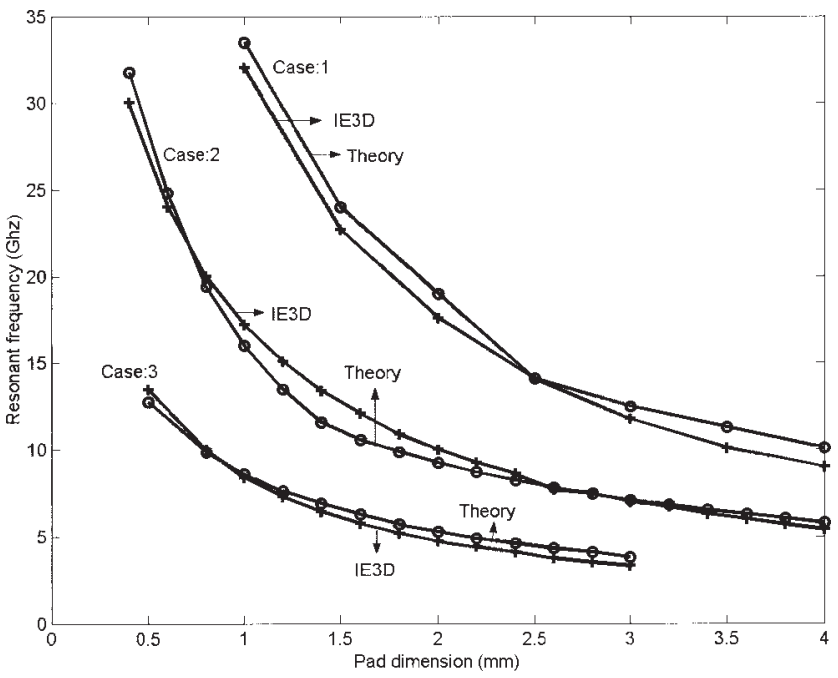

Figure 3 Series (short circuit) resonance frequencies computed using this theory and IE3D

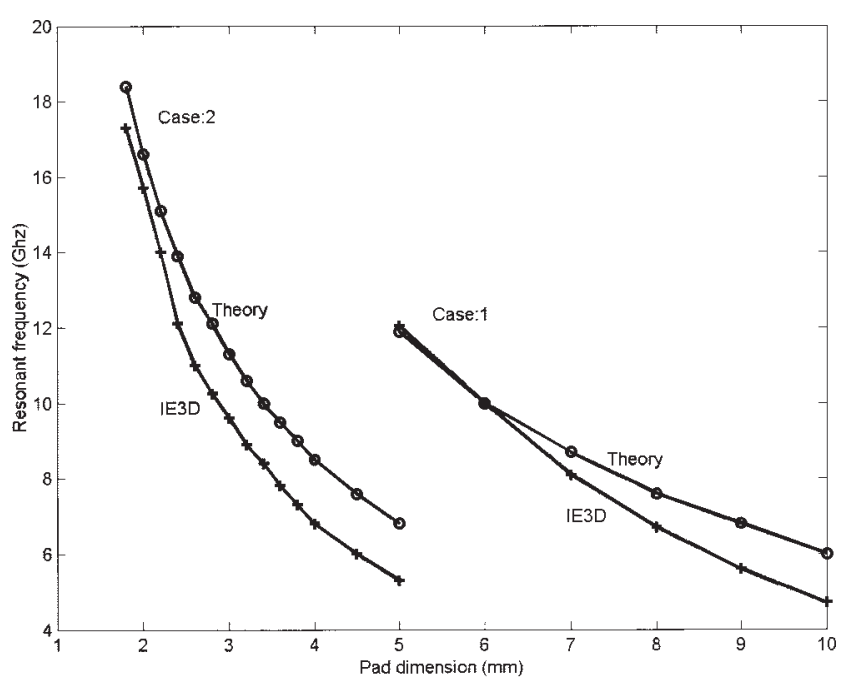

Figure 4 Parallel (open circuit) resonance frequencies computed using this theory and IE3D

In this paper, a coupling pad and the pin-pad resonators described above are used to improve the stopband performance by creating transmission zeros as well as suppressing the $2^{\text {nd }}$ harmonic of a filter. The proposed filter configuration is shown in Figure 5.

Figures 6 and 7 show the responses of three different filter designs. First, the conventional approach (dashed lines) contains neither the coupling pad nor pin-pad resonators. The passband at the $3^{\text {rd }}$ harmonic is visible at around $2.5 \mathrm{GHz}$. Next, we introduce two different pin-pad resonators (dashed-dotted lines), which are designed to suppress the second passband by choosing the pin and pad dimensions such that their resonant frequencies coincide with that passband. The respective transmission zeros due to the two pin-pad resonators are denoted as Tz:3 and Tz:4, as shown in Figure 6 .

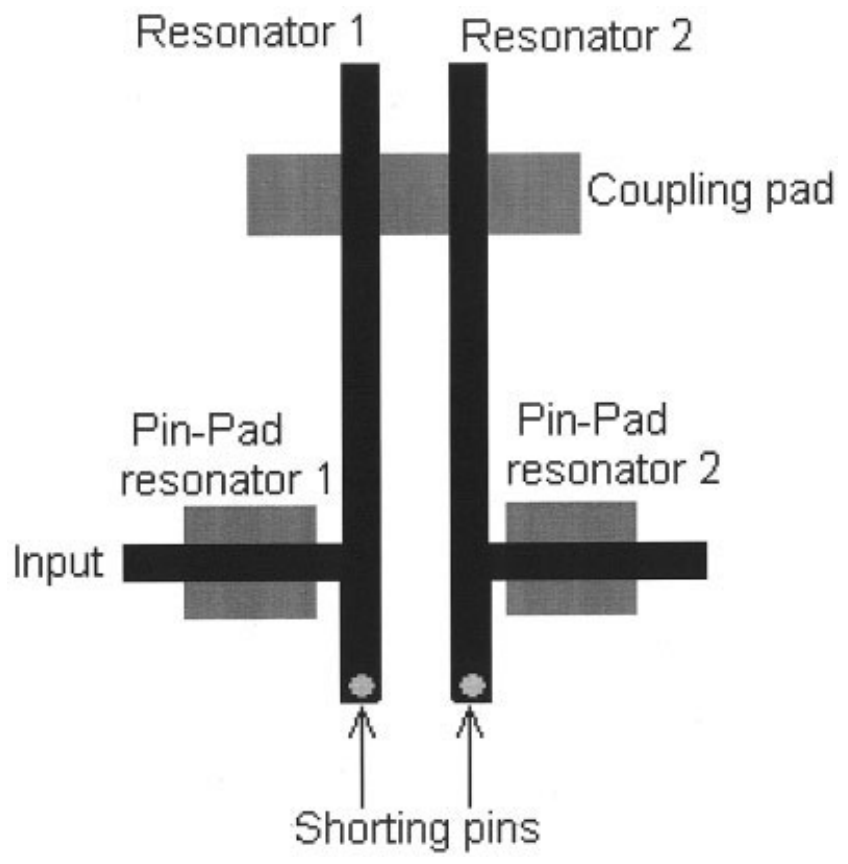

Figure 5 Proposed filter configuration 


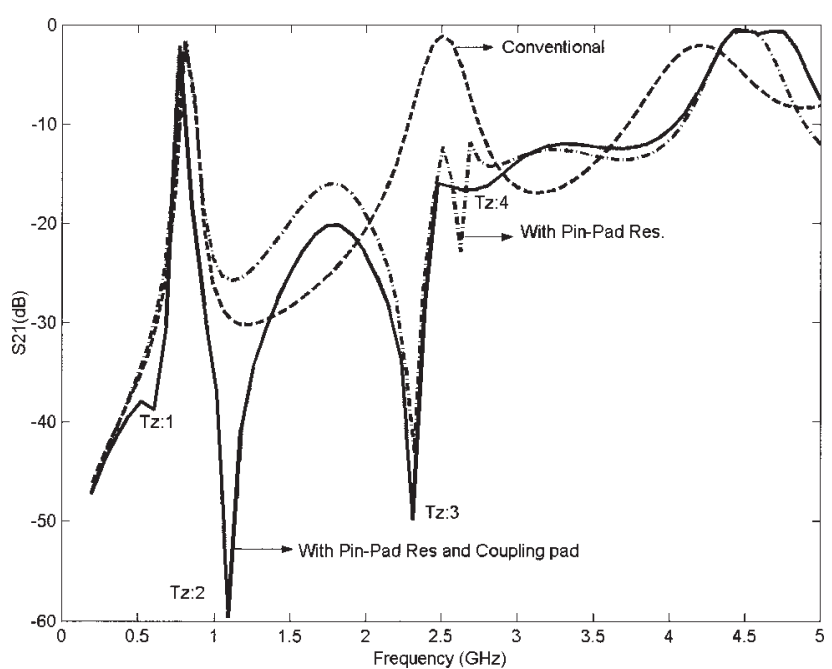

Figure 6 Transmission performance in $\mathrm{dB}$ of different filter configurations (IE3D). Conventional design without pin-pad resonators or coupling pad (dashed line); adding pin-pad resonators (dash-dotted line); coupling pad and pin-pad resonators (solid line)

Finally, we employ the coupling pad (see also Fig. 5) to create two more transmission zeros (solid lines in Figs. 6 and 7). The first transmission zero (Tz:1) is due to the parallel resonance that is formed by two components: (i) the electromagnetically coupled signal created between the resonators and (ii) the signal through the coupling pad. The second transmission zero (Tz:2) is due to the possible resonance through the fringe capacitance and inductance of the coupling pad with resonators and the ground plane. The dimensions of the various sections of the filter with coupling pad and pin-pad resonators are as follows: $\varepsilon_{r}=7.5, h=s+l=0.5$ $\mathrm{mm}, W=0.6 \mathrm{~mm}$; resonators: length $=40 \mathrm{~mm}$, spacing between the resonators $=1 \mathrm{~mm}$, input/output line location $=4.5 \mathrm{~mm}$ from short-circuit end; pin-pad resonator 1: $x=y=7.25 \mathrm{~mm}, l=$ $0.375 \mathrm{~mm}, d=0.1 \mathrm{~mm}$; pin-pad resonator $2: x=y=8.6 \mathrm{~mm}$, $l=0.375 \mathrm{~mm}, d=0.1 \mathrm{~mm}$; coupling pad: length (along resonators) $=4 \mathrm{~mm}$, width $=3 \mathrm{~mm}$.

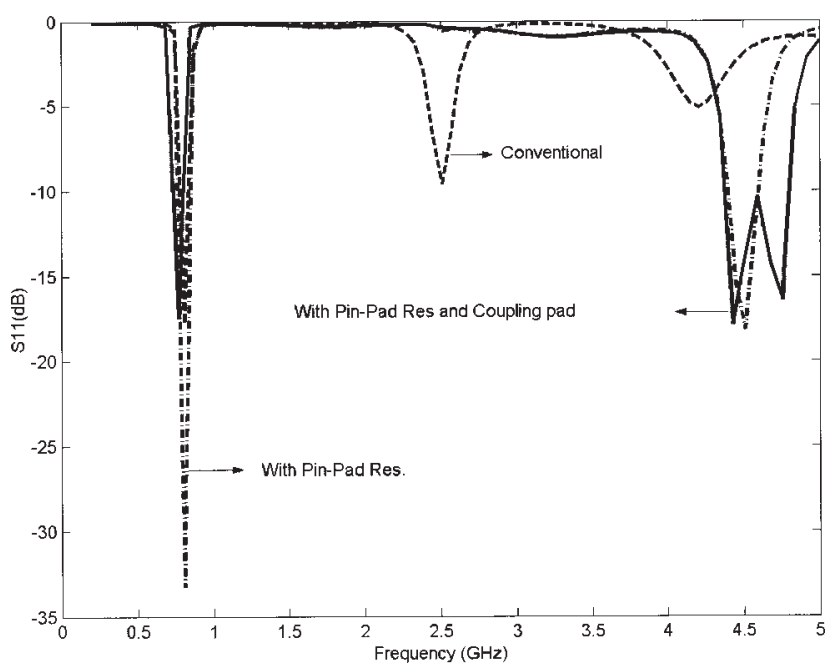

Figure 7 Reflection performance in $\mathrm{dB}$ of different filter configurations (IE3D). Conventional design without pin-pad resonators or coupling pad (dashed line); adding pin-pad resonators (dash-dotted line); coupling pad and pin-pad resonators (solid line)
Note that the results presented in Figures 6 and 7 have been obtained using IE3D ${ }^{\odot}$ and, therefore, include all electromagnetic effects.

\section{CONCLUSION}

The equivalent-circuit model for grounded pin-pad resonators offers an attractive solution for the creation of transmission zeros in LTCC filter components. The determination of the resonance frequency or, alternatively, the pin-pad design for a given frequency is simple and straightforward. The process has been validated through an LTCC filter design, which was shown to achieve a transmission zero per pin-pad resonator. Hence, this design can be used to suppress a second passband. An additional coupling pad can be used to create additional transmission zeros closer to the passband. Both the equivalent-circuit model and the LTCC filter designs were verified through computations using the commercially available field solver $\mathrm{IE}^{\circ}{ }^{\odot}$.

\section{REFERENCES}

1. D.F. Sievenpiper, L. Zhang, F.J. Broas, N.G. Alexopoulos, and E. Yablonovitch, High-impedance electromagnetic surfaces with a forbidden frequency band, IEEE Trans Microwave Theory Tech 47 (1999), 2059-2074.

2. Y. Horii, A compact microstrip patch antenna having a 2-dimensional grounded-pad array embedded in an LTCC substrate, IEEE AP-S Int Symp, Columbus, OH, 2003, pp. 986-989.

3. Y. Horii, Filtering effects of grounded patches embedded in a microstrip line substrate, IEICE Jpn Tech Rpt MWP02-3 (2002), 15-22.

4. B.C. Wadell, Transmission line design handbook, Artech House, Boston, 1991.

5. G. Matthaei, L. Young, and E.M.T. Jones, Microwave filters, impedance matching networks and coupling structures, Artech House, Boston, 1980.

6. L.H. Hsieh and K. Chang, Compact elliptic function lowpass filters using microstrip stepped impedance hairpin resonators, IEEE Trans Microwave Theory Tech 51 (2003), 193-199.

7. T. Ishizaki, M. Fujita, H. Kagata, T. Uwano, and H. Miyake, A very small dielectric planar filter for portable telephones, IEEE Trans Microwave Theory Tech 42 (1994), 2017-2022.

8. K. Rambabu and J. Bornemann, Simplified analysis technique for the initial design of a class of LTCC filters, IEEE Trans Microwave Theory Tech 53 (2005), 1787-1791.

(C) 2005 Wiley Periodicals, Inc.

\section{DESIGN OF PIN DIODE CONTROLLED VARIABLE ATTENUATOR USING SLOW WAVE MICROSTRIP LINES}

\author{
Kae-Oh Sun and Daniel van der Weide \\ Department of Electrical and Computer Engineering \\ University of Wisconsin \\ 1415 Engineering Drive, Madison, WI
}

Received 18 May 2005

ABSTRACT: We describe a simple PIN-diode-controlled variable attenuator that employs a $0-d B$ branch-line directional coupler, and present a method to reduce the size using slow-wave microstrip lines. At the center frequency, the attenuation monotonically varied from 0.7 to $23 \mathrm{~dB}$ with the control voltage. By using slow-wave microstrip lines, we achieve a $60 \%$ size reduction compared to a conventional structure.@ 2005 Wiley Periodicals, Inc. Microwave Opt Technol Lett 47: 323-327, 2005; Published online in Wiley InterScience (www. interscience.wiley.com). DOI 10.1002/mop.21159 